\title{
Erratum
}

\section{Erratum: COVID-19 Molecular Testing in Korea: Practical Essentials and Answers From Experts Based on Experiences of Emergency Use Authorization Assays}

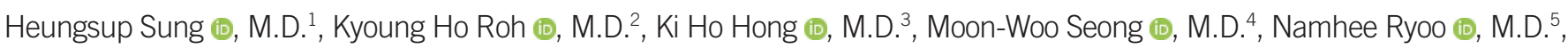

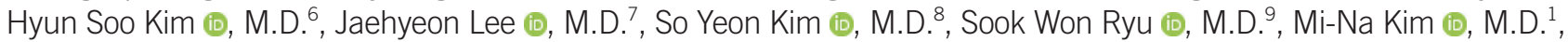
Myung Guk Han (1), D.V.M. ${ }^{10}$, Sang Won Lee $\mathbb{0}$, Ph.D..$^{10}$, Hyukmin Lee $\left(\mathbb{0}\right.$, M.D. $^{11}$, and Cheon Kwon Yoo $\mathbb{1}$, Ph.D. ${ }^{10}$; on behalf of COVID-19 Diagnosis Test Management Committee established by Korea Centers for Disease Control and Prevention

${ }^{1}$ Department of Laboratory Medicine, Asan Medical Center and University of Ulsan College of Medicine, Seoul, Korea; ${ }^{2}$ Department of Laboratory Medicine, National Health Insurance Service, Ilsan Hospital, Goyang, Korea; ${ }^{3}$ Department of Laboratory Medicine, Seoul Medical Center, Seoul, Korea; ${ }^{4}$ Department of Laboratory Medicine, Seoul National University Hospital, Seoul, Korea; ${ }^{5}$ Department of Laboratory Medicine, Keimyung University School of Medicine, Daegu, Korea; ${ }^{6}$ Department of Laboratory Medicine, Hallym University College of Medicine, Chuncheon, Korea; ${ }^{7}$ Department of Laboratory Medicine, Jeonbuk National University Medical School and Hospital, Jeonju, Korea; ${ }^{8}$ Department of Laboratory Medicine, National Medical Center, Seoul, Korea; ${ }^{9}$ Department of Laboratory Medicine, Kangwon National University School of Medicine, Chuncheon, Korea; ${ }^{10}$ Center for Laboratory Control of Infectious Diseases, Korea Centers for Disease Control and Prevention, Osong, Korea; ${ }^{11}$ Department of Laboratory Medicine, Yonsei University College of Medicine, Seoul, Korea

This erratum corrects an error in an author's name in the article entitled "COVID-19 Molecular Testing in Korea: Practical Essentials and Answers From Experts Based on Experiences of Emergency Use Authorization Assays" by Heungsup Sung, Kyoung Ho Roh, Ki Ho Hong, Moon-Woo Seong, Namhee Ryoo, Hyun Soo Kim, Jaehyeon Lee, So Yeon Kim, Sookwon Yoo, Mi-Na Kim, Myung Guk Han, Sang Won Lee, Hyukmin Lee, and Cheon Kwon Yoo on behalf of COVID-19 Diagnosis Test Management Committee established by Korea Centers for Disease Control and Prevention in Ann Lab Med 2020;40:439-447 (https://doi.org/10.3343/ alm.2020.40.6.439).

\section{Before correction:}

Page 439:

Heungsup Sung, M.D., Kyoung Ho Roh, M.D., Ki Ho Hong, M.D., Moon-Woo Seong, M.D., Namhee Ryoo, M.D., Hyun Soo Kim, M.D., Jaehyeon Lee, M.D., So Yeon Kim, M.D., Sookwon Yoo, M.D., Mi-Na Kim, M.D., Myung Guk Han, D.V.M., Sang Won Lee, Ph.D., Hyukmin Lee, M.D., and Cheon Kwon Yoo, Ph.D.; on behalf of COVID-19 Diagnosis Test Management Committee established by Korea Centers for Disease Control and Prevention

Corresponding author: Heungsup Sung, M.D.

Department of Laboratory Medicine, Asan Medical Center and University of Ulsan College of Medicine, 88 Olympic-ro 43-gil, Songpa-gu, Seoul 05505, Korea

Tel: +82-2-3010-4499, Fax: +82-2-478-0884, E-mail: sung@amc.seoul.kr

Co-corresponding author: Cheon Kwon Yoo, Ph.D.

Center for Laboratory Control of Infectious Diseases, Korea Centers for Disease Control \& Prevention, Osong Health Technology Administration Complex, 187 Osongsaengmyeong 2-ro, Osong-eup, Heungdeok-gu, Cheongju 28159, Korea

Tel: +82-43-719-8100, Fax: +82-43-719-8149, E-mail: ckyoo@korea.kr

\section{(c) (i) (2)}

(c) Korean Society for Laboratory Medicine

This is an Open Access article distributed under the terms of the Creative Commons Attribution Non-Commercial License (https://creativecommons.org/licenses/by-nc/4.0) which permits unrestricted non-commercial use, distribution, and reproduction in any medium, provided the original work is properly cited. 
Sung $\mathbf{H}$, et al.

Practical essentials regarding COVID-19 molecular testing
ANNALS OF

LABORATORY MEDICINE

\section{After correction:}

Page 439:

Heungsup Sung, M.D., Kyoung Ho Roh, M.D., Ki Ho Hong, M.D., Moon-Woo Seong, M.D., Namhee Ryoo, M.D., Hyun Soo Kim, M.D., Jaehyeon Lee, M.D., So Yeon Kim, M.D., Sook Won Ryu, M.D., Mi-Na Kim, M.D., Myung Guk Han, D.V.M., Sang Won Lee, Ph.D., Hyukmin Lee, M.D., and Cheon Kwon Yoo, Ph.D.; on behalf of COVID-19 Diagnosis Test Management Committee established by Korea Centers for Disease Control and Prevention 